\title{
A Neural Recording and Stimulation Chip with Artifact Suppression for Biomedical Devices
}

\author{
Xu Liu $\mathbb{D}^{1}{ }^{1}$ Juzhe Li $\left(\mathbb{D},{ }^{1}\right.$ Tao Chen, ${ }^{2}$ Wensi Wang $\left(\mathbb{D},{ }^{3}\right.$ and Minkyu Je $\mathbb{D}^{4}$ \\ ${ }^{1}$ College of Microelectronics, Beijing University of Technology, Ping Le Yuan 100\# Chao Yang District, Beijing, China \\ ${ }^{2}$ Advanced Photonics Institute, Beijing University of Technology, Ping Le Yuan 100\# Chao Yang District, Beijing, China \\ ${ }^{3}$ Engineering Research Center of Intelligent Perception and Autonomous Control (Ministry of Education), \\ Beijing University of Technology, Ping Le Yuan 100\# Chao Yang District, Beijing, China \\ ${ }^{4}$ Korea Advanced Institute of Science and Technology, 291 Daehak-ro Yuseong-Gu, Daejeon, Republic of Korea
}

Correspondence should be addressed to Xu Liu; liuxu16@bjut.edu.cn

Received 24 June 2021; Accepted 18 August 2021; Published 27 August 2021

Academic Editor: Kheng-Lim Goh

Copyright (C) $2021 \mathrm{Xu}$ Liu et al. This is an open access article distributed under the Creative Commons Attribution License, which permits unrestricted use, distribution, and reproduction in any medium, provided the original work is properly cited.

\begin{abstract}
This paper presents chip implementation of the integrated neural recording and stimulation system with stimulation-induced artifact suppression. The implemented chip consists of low-power neural recording circuits, stimulation circuits, and action potential detection circuits. These circuits constitute a closed-loop simultaneous neural recording and stimulation system for biomedical devices, and a proposed artifact suppression technique is used in the system. Moreover, this paper also presents the measurement and experiment results of the implemented 4-to-4 channel neural recording and stimulation chip with $0.18 \mu \mathrm{m}$ CMOS technology. The function and efficacy of simultaneous neural recording and stimulation is validated in both in vivo and animal experiments.
\end{abstract}

\section{Introduction}

The neural prosthesis system includes a neural/muscular stimulator and neural recording circuit. These stimulators and recorders of the system have been widely used in many medical fields for decades, such as cochlear/retinal prosthesis, cell activation, and cardiac pacemaker [1-5]. Functionally, neural stimulation is used to activate the prosthesis and wake up the sensory function [6], while neural recording can sense nerve signals or complete the evaluation of stimulation effect [7-9]. Combining neural stimulator and neural recorder, the closed-loop controlled simultaneous neural recording and stimulation system is formed to recover the basic functions of the injured individuals [10-16], such as the system for epileptic seizure detection and suppression [17, 18].

As shown in Figure 1, in the closed-loop neural recording and stimulation system for epileptic seizure detection and suppression, neural recording is used to detect the epileptic signals in brain, and electrical stimulation is used to suppress epileptic seizures. However, the electrical stimulation pulse will cause artifacts and prevent the recording amplifier from normal operation, so the closed-loop system cannot make timely and correct sense-and-trigger response. The problem of stimulation artifact also affects the function of the system in other biomedical devices for brain stimulation and recording [20-22].

1.1. Stimulation-Induced Artifact in the Closed-Loop System. To realize multiple functions in biomedical equipment, the neural/muscular recording and stimulation system generally includes multiple recording and stimulation channels, with recording circuits, data processing circuits, stimulation circuits, and electrodes. A bipolar stimulation system is shown in Figure 2(a), which includes a recording circuit with an electrode and a stimulator with one working electrode and one reference electrode. The stimulator with the working electrode provides bidirectional and well-matched stimulation current. During the stimulation, biphasic 


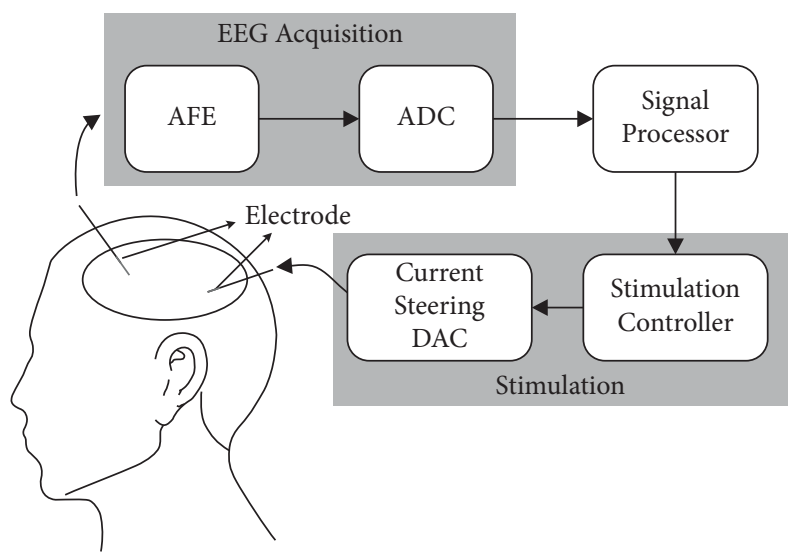

FIGURE 1: Concept of the system for epileptic seizure detection and suppression.

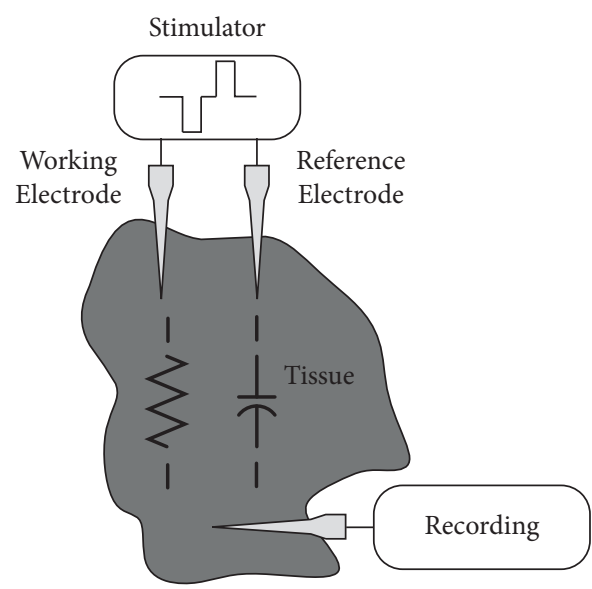

(a)

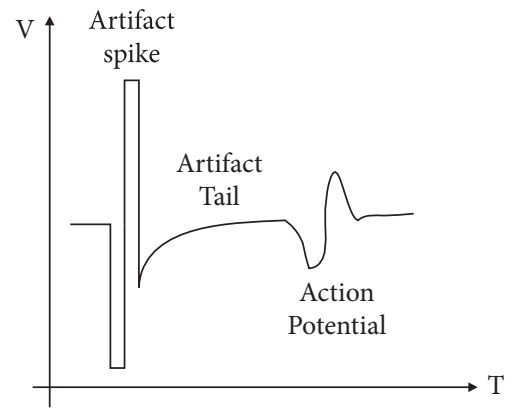

(b)

Figure 2: (a) Simplified interface between a bidirectional current stimulator and a signal recorder. (b) The output voltage waveform of the recording front end.

current flows through the tissue between two electrodes. Therefore, the voltage variation emerges at the stimulation site near the working electrode due to its resistive and capacitive feature. The amplitude of this voltage variation usually ranges from a few hundred millivolts to a few volts, which is mainly determined by the current level and the electrode impedance [23-26]. Then, the voltage waveform generated at the stimulation site has transmitted to the neural recording front end (RFE) through tissue, and it saturates the high-gain recording amplifier. The output signal from the saturated amplifier is called stimulationinduced artefact. The amplifier usually takes a long time to recover from this undesired saturation $[23,24]$.

Figure 2(b) shows the output voltage waveform of the recording front end, which includes an artifact spike, an artifact tail, and an action potential (AP). This AP has been recorded after the unexpected artifact spike. When the artifact happens, the RFE generally needs $2-10 \mathrm{~ms}$ to fully recover and be ready to record the next action potential [27]. Thus, neural recording cannot work normally within this duration, and the next action potential can only be observed after the recording amplifier is fully recovered. Such an artifact problem can be found in most simultaneous recording and stimulation systems $[14,15,19,28-30]$.

1.2. Stimulation Artifact Suppression. Several artifact suppression techniques have been reported previously. In the blanking technique $[15,31-34]$, the RFE is switched off during the stimulation period and turned on during the neural recording. However, the neural signals during the "blanking" period cannot be recorded. In the signal postprocessing technique [35-39], neural signal can be recovered by subtracting the artifact template from the recorded signal. The advantage of the digital processing method is that no neural signal is missed during neural recording. But it is computationally intensive and requests RFE to have a very large dynamic range (several volts) to record artifact signal. In order to obtain a large input dynamic range to record the artifact signal, a track-and-zoom ADC is proposed in [40]. The dynamic input range of RFE is exponentially expanded with the recording signal, which prevents the saturation of 
neural recording. Another method is to replace the amplifier with a voltage-controlled oscillator (VCO) [41]. This method eliminates the problem of artifact-induced saturation of the amplifier. But the VCO needs further noise optimization to improve the signal-to-noise ratio of neural recording.

Another technique reported is localized stimulation $[42,43]$. In this method, the biphasic current can flow through the tissue and return to a local ground. This reduces the artifact amplitude and allows the amplifier to quickly recover to the normal recording state, but the artifact is still not well suppressed. An improved method is dual electrode in-phase stimulation, which carries out differential acquisition at the recording amplifiers [44]. This method has good artifacts suppression effect, but it requires strict impedance matching among the recording electrodes. It is desired to design an extra accurate impedance matching network.

In this paper, we present our implemented closed-loop neural recording and stimulation chip with an artifact cancellation technique. Using the referenced and tuned push-pull stimulation (RTPPS) scheme with a tripolar electrode, the problem of artifact can be solved. A tripolar stimulation configuration with two working electrodes and one reference electrode are used in our method. The stimulation currents delivered by two working electrodes are complementary to each other. By doing so, the impact of large stimulation voltage fluctuation propagated to the recording site can be significantly reduced. The proposed concept is demonstrated with a prototype system including four recording channels and four stimulation channels, in both in vitro and in vivo experiments.

The rest of the paper is organized as follows. Section 2 explains the proposed stimulation artifact suppression technique and describes the implementation of the prototype recording and stimulation system. Section 3 presents the bench-top measurement results, as well as the in vitro and in vivo experiment results. Conclusion is given in Section 4 .

\section{Implemented Closed-Loop Neural Prosthesis System with Artifact Cancellation}

2.1. 4-Channel Neural Recording and Stimulation Chip Implementation. A 4-channel neural recording and stimulation system is designed and presented in this work. Figure 3 shows the system block diagram. The system consists of four-channel RFEs, four action potential detectors (APD), a global digital (DIG) control block, power down (PD) control, bandgap reference block (BGB) for biasing circuit, and four high-voltage artifact-suppressed stimulators (HVAS). The system can be configured either for multichannel neural recording applications using RFE channels or multichannel neural/muscular stimulation using HVAS channels. With both RFE and HVAS channels active, the system can be configured in four modes: recording (REC), stimulation (STIM), closed-loop recording-stimulation (REC-STIM), and stimulation-recording (STIM-REC). In the REC-STIM mode, the system performs neural signal recording, action potential detection, and action-potential-triggered stimulation. In STIM-REC mode, the stimulator generates stimulation pulses for the specific muscles or neurons, while the RFE is used to monitor stimulation invoked neural signals. In all stimulation-related modes, passive charge balancing (PCB) circuit is used to remove the residual charge after each stimulation pulse to prevent tissue damage. As shown in Figure 3, the PCB circuit is a passive path between the stimulating electrodes and the ground, controlled by CMOS switches. The PCB is only activated at the end of each stimulation pulse.

An artifact cancellation scheme is used in this system design. The so-called RTPPS scheme mentioned in Section 1 aims to cancel the artifact at the stimulation site so that the artifact will not affect the recording site. In conventional bipolar stimulation configuration, the biphasic current (cathodic-then-anodic) flows from a working electrode to a reference electrode. As a result, the stimulation current causes a voltage change at the interface of the working electrodes. This voltage signal is coupled to the input of RFE through tissue and causes artifact. In our implemented stimulator [45], two working electrodes (WE) and one reference electrode (RE) are used to form a tripolar electrode. In this case, the second working electrode counteracts the stimulation from the first working electrode and thus cancels the coupling from the stimulation site to the input of the recording front end. The stimulation currents for the two electrodes are generated by two current generators, namely, the stimulation current generator (SCG) and the countercurrent generator (CCG). By using such stimulation scheme, this system eliminates the voltage fluctuation on the stimulation site and hence reduces the stimulation induced artifact.

2.2. High-Voltage Artifact-Suppressed Stimulator. Figure 4 shows the circuit schematic of one stimulator channel, which includes two 10-bit current steering digitalto-analog converters (DACs) and two high-voltage current drivers (HVCD). SCG and CCG have a similar structure and are composed of one DAC and one HVCD. The SCG and CCG produce the same biphasic currents in amplitude to the stimulation target through the electrodes with identical impedance, but with inversed phase. The stimulation pulse width is determined by the timing control of the signals cathodic and anodic in high-voltage current drivers. In the neural stimulator based on constant current stimulation (CCS), the voltage on the electrode depends on the characteristic impedance of the electrode-tissue interface. For larger impedance, HVCD is required. HVCD contains current mirrors with a ratio of 10 to amplify the output current from DAC. The MOS switches connected to the transistors gates are used to activate or deactivate the current generator. To reduce the chip power consumption, the DAC is powered by a $1.8 \mathrm{~V}$ supply, and the HVCD is powered by $24 \mathrm{~V}$. The output voltage compliance is therefore large to deliver sufficient stimulation current. The reference voltage is set to a half VDD_h. The current amplitudes of both SCG and CCG are set by DACs and controlled by digital blocks. Besides, the amplitudes of the currents from CCG and SCG are made tunable to compensate for any mismatch between the two electrode interfaces. 


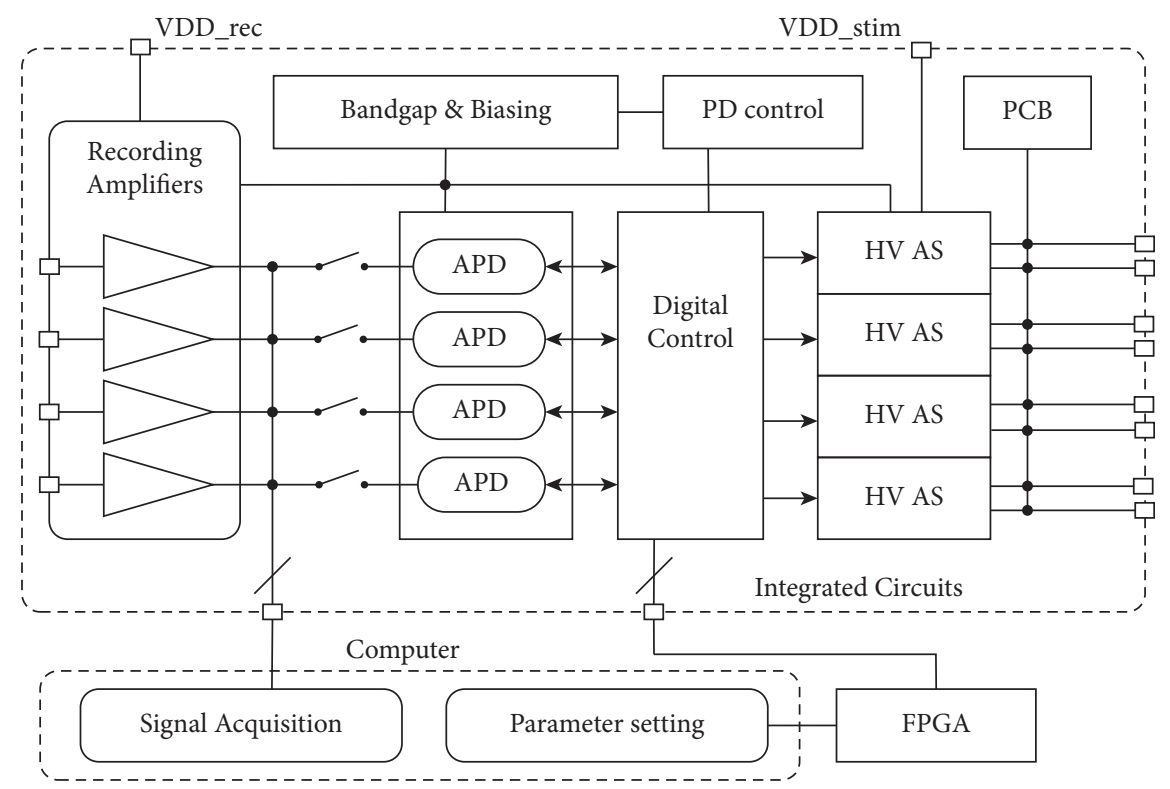

FIGURE 3: System block diagram of 4-channel neural recording/stimulation system.

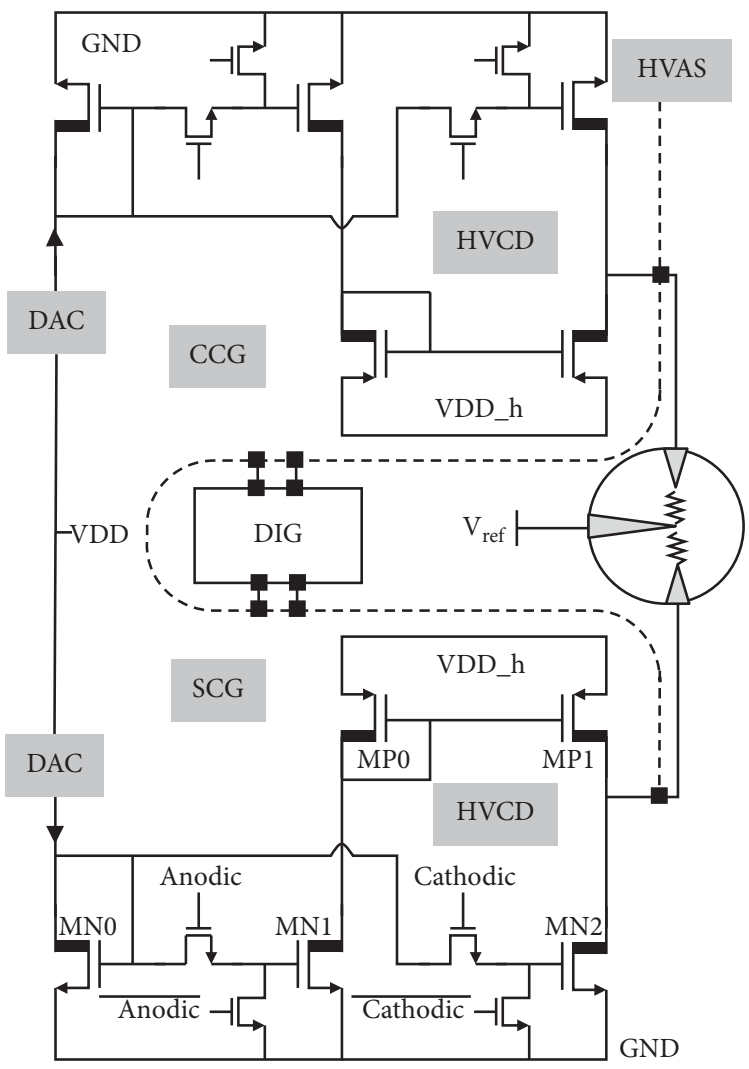

FIGURE 4: Schematic of high-voltage artifact-suppressed stimulator (in each channel).

2.3. Recording Front End and Action Potential Detector. The neural amplifier is one of the most important parts in the neural prosthesis system. As shown in Figure 5(a), the neural recording front end consists of a neural amplifier, a band pass filter (BPF), and a buffer. The capacitor feedback topology with pseudoresistance is selected to achieve low power consumption and low noise. Since the low-pass cutoff frequency of the recording system is determined by the $\mathrm{BPF}$, the bandwidth of the RFE is set to be slightly larger than the nerve signal bandwidth. The gain of the amplifier can be 

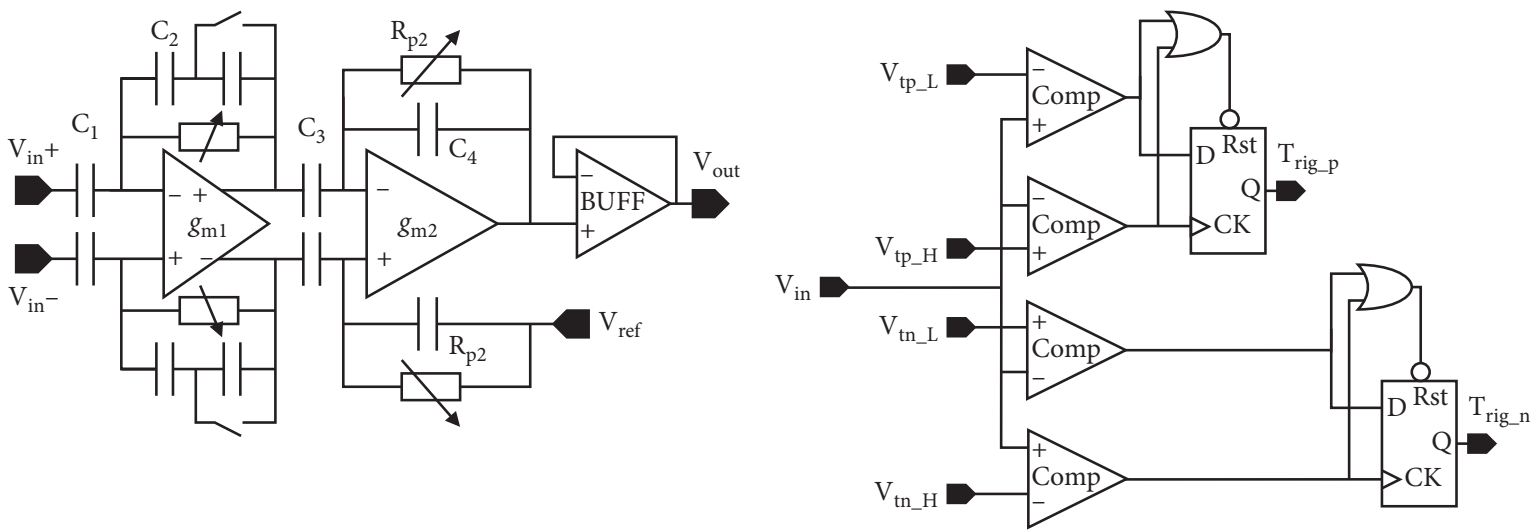

(a)

(b)

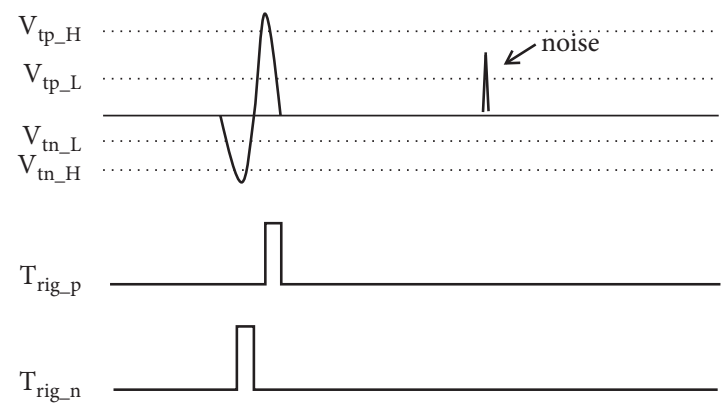

(c)

Figure 5: (a) Neural recording front-end (RFE) circuit. (b) Action potential detector circuit. (c) Signal waveforms for functionality illustration of the circuit.

adjusted by the ratio of input capacitance to feedback capacitance. RFE has a programmable gain of $54 / 60 \mathrm{~dB}$. The high- and low-pass cut-off frequencies can be programmed for different recording modes (spikes only, LPF only, or both). The details of the amplifier design in RFE are described in $[46,47]$.

The APD is a simple threshold detector, as shown in Figure 5(b). It contains four comparators and two flip-flops (FF). The APD detects both positive and negative spikes, depending on which comes first. The upper 2 comparators and FF are to detect positive spikes. The threshold voltage levels can be tuned by Vtp_H and Vtp_L, and the output trigger signal is asserted when the amplitude of the spike exceeds Vtp_H and becomes nil when the amplitude of the spike drops below Vtp_L as shown in Figure 5(c). This hysteresis window (between Vtp_H and Vtp_L) provides some noise immunity to the detector. Similarly, the lower two comparators and FF are used to detect negative spikes. The hysteresis window can be tuned by Vtn_L and Vtn_H. The trigger signal generated from APD is sent to the digital control block of the system which controls the stimulator. With these two triggering signals from positive and negative threshold detectors, simple spike pattern recognition such as distinguishing biphasic spikes from electrical glitches is enabled.

2.4. Digital Control Block. The main functions of the digital control block are to set stimulation parameters and control the stimulation. The digital block has a default command which determines the stimulation parameters such as amplitude and duration. These parameters can also be programmed by an external FPGA through a serial command. The command format, control timing, and function flow of the digital block are shown in Figure 6(a).

Stimulation parameters are decoded from the command frame and stored in global digital control registers. The cathodic and anodic stimulation pulse width ( $T 1$ and $T 2$ ) use 8-bit control, respectively, and the interphasic delay (Tint) between the two stimulation phases is in 10-bit control. The stimulation current amplitude (VGCM1/VGCM2) control is also in 10-bit. In a typical scenario, several command frames are sent first to configure stimulation parameters. When a spike signal is detected by APD, the embedded finite state machine (FSM) generates control signals such as $b s / c<0$ : $4>$, Iin_s/c $\langle 0: 4>$, cathodic, anodic, and idle to deliver a predefined biphasic stimulus. After each stimulation pulse, the external switches connect electrodes WE1, WE2, and RE during the idle phase for passive charge balancing (PCB), as shown in Figure 6(b). The interphasic delay between cathodic and anodic phases can be programmed in the range from $0 \mu$ s to $255 \mu$ s. The detailed stimulation control timing and output waveforms are shown in Figure 6(c). For arbitrary stimulation waveform generation, the pulse width of cathodic and anodic stimulation is divided into 16 steps, respectively, as shown in Figure 6(d). As a result, the current amplitude (D1'-D16', D1-D16) of each step can be set by sending different commands to the DAC registers. 


\begin{tabular}{|c|c|c|}
\hline \multirow{2}{*}{ Header } & \multirow{2}{*}{ Addr } & CMD value \\
\cline { 3 - 3 } & & T1/T2/ $\mathrm{T}_{\text {int }} /$ Range/VGCM1/VGCM2 \\
\hline 12bit & 8bit & $8 \sim 80 \mathrm{bit}$ \\
\hline
\end{tabular}

(a)

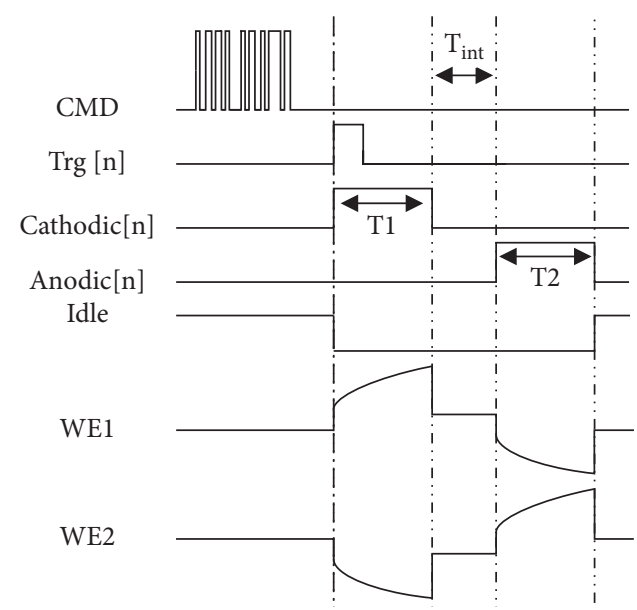

(c)

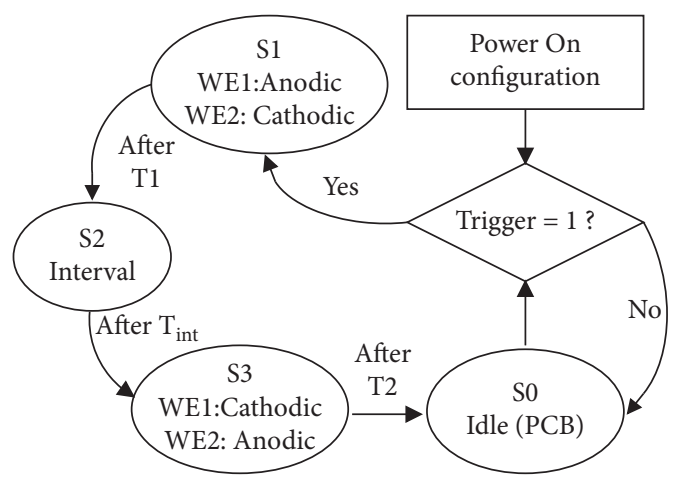

(b)

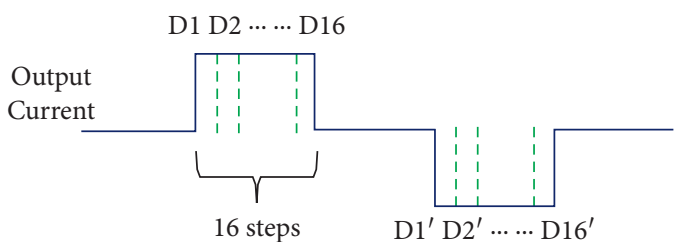

(d)

Figure 6: (a) Command frame. (b) State machine for stimulator control. (c) Timing and voltage waveforms of control and output signals. (d) Stimulation pulse width division for arbitrary waveform control.

\section{Measurement Results}

3.1. Bench-Top Measurement Results. The 4-to-4-channel closed-loop neural recording and stimulation system is implemented in $0.18-\mu \mathrm{m}$ high-voltage CMOS technology with LDMOS option. The chip microphotograph is shown in Figure 7. The total chip area is $2 \mathrm{~mm}$ by $2 \mathrm{~mm}$. Figure 8 (a) shows the measured output waveforms of two independent HVAS channels of the system. In each channel, the cathodic and anodic current amplitudes are set by two independent 10-bit DACs. It can generate arbitrary stimulation current waveforms including exponential, triangular, ramp, and constant waveforms, respectively, depending on the application requirement. Pulse durations $T 1$ and $T 2$ are also adjustable in the range from $16 \mu$ s to $4 \mathrm{~ms}$.

Figure 8 (b) demonstrates test results of the chip configured in the REC-STIM mode. In this configuration, the recording electrodes of RFE and the stimulation electrodes of HVAS are electrically isolated. An ECG signal generated from a function generator is used as a dummy neural action potential signal to the input of one RFE channel. The amplitude of ECG pulse is set as $800 \mu \mathrm{V}$ peak-to-peak with a frequency of $100 \mathrm{~Hz}$. The gain of RFE is set as $60 \mathrm{~dB}(1000 \mathrm{~V} / \mathrm{V})$. The band-pass filter of RFE is turned off. As shown in Figure 8(c), the APD detects the output of RFE and generates the trigger signal for HVAS.
Biphasic stimulation pulses are generated by HVAS and delivered to the electrode nodes, namely, WE1, WE2, and RE. The current waveform is configured as constant current of $600 \mu \mathrm{A}$ with a pulse duration of $320 \mu$ s. The reference voltage on RE is set at $12(\mathrm{~V})$. A dummy load (a $10 \mathrm{k} \Omega$ resistor and a $100 \mathrm{nF}$ capacitor in series) is used between each stimulation output and the reference voltage source.

To demonstrate the proposed artifact-suppression technique, an experiment is done with recording and stimulation electrodes in phosphate-buffered saline (PBS) as shown in Figure $8(\mathrm{c})$. In the REC-STIM mode, one recording channel is used to record the ECG signal when the ECG spike is detected the stimulator is triggered, delivering the stimulation current to the PBS solution. It is observed that when CCG is disabled, the stimulation artifact saturates the output of the recording amplifier, as shown in Figure 8(d), which needs a long period of time to recover to the normal state before it can perform recording again. However, with CCG enabled in Figure 8(e), an intact ECG signal is recorded during the stimulation period and the saturation is not observed at the output of the recording amplifier.

3.2. Animal Experiment. Two in vivo experiments on the rat are carried out using the implemented neural recording and 


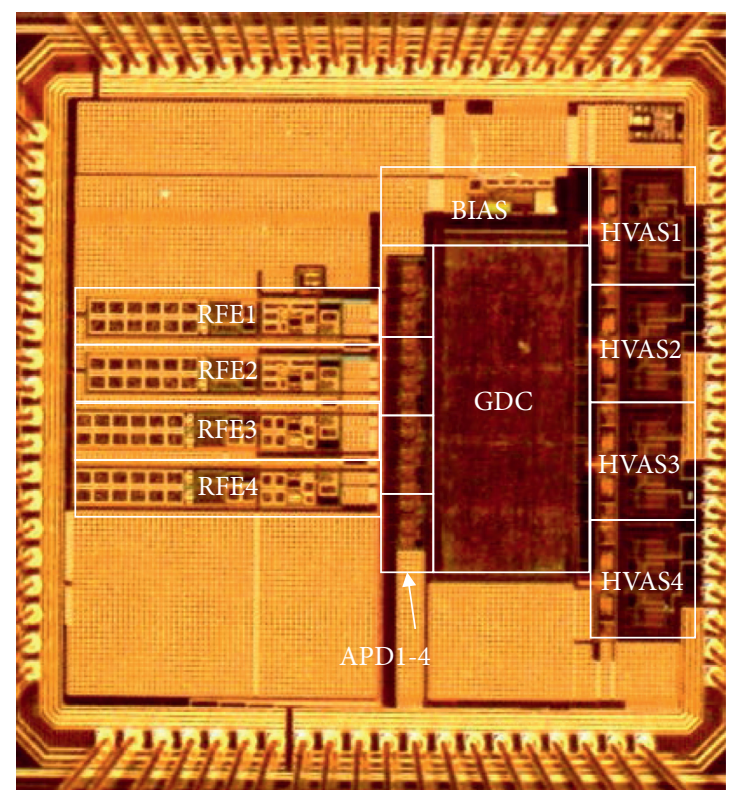

Figure 7: 4-channel recording/stimulation IC microphotograph.

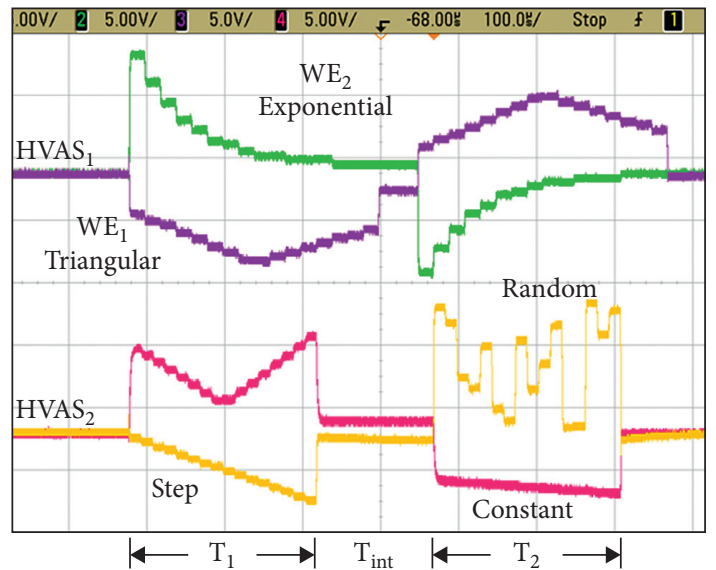

(a)

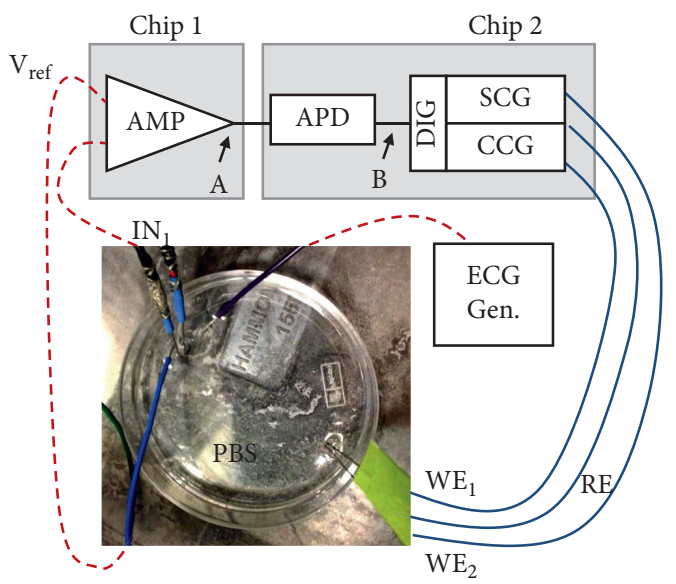

(c)

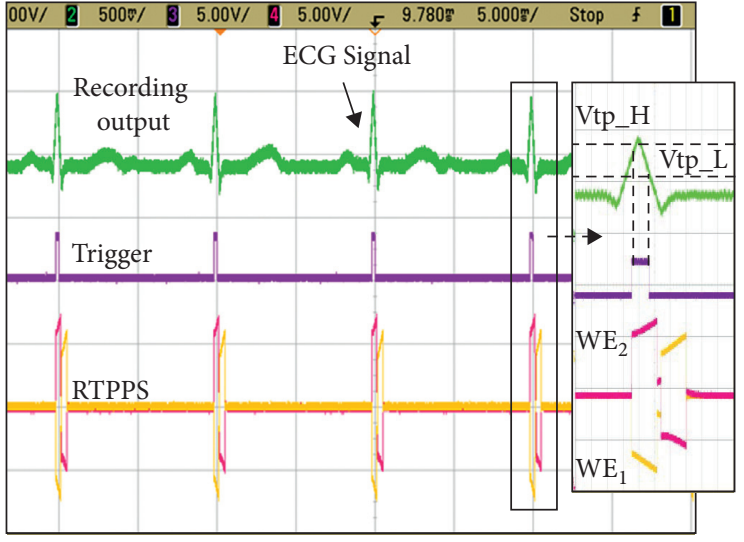

(b)

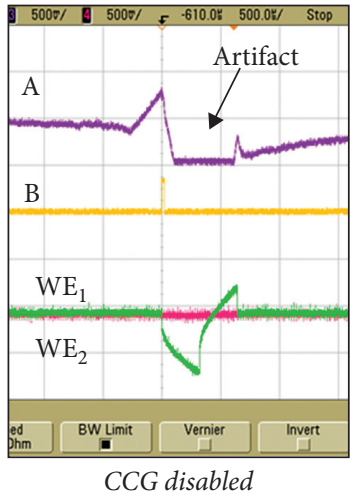

(d)

FIgUre 8: Continued. 


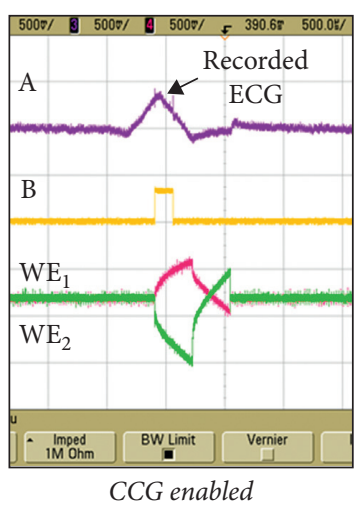

(e)

Figure 8: (a) Arbitrary stimulation waveforms from two HVAS channels. (b) Test-bench measurement results on one channel: output waveforms of recording circuit, APD, and the HVAS stimulator. (c) In vitro test setup and (d, e) Measurement results with and without RTPPS: the top and middle traces show recording outputs from REF channels 1 and 2, respectively. The bottom two traces are the measured voltages on two working stimulation electrodes.

stimulation system. The first experiment is to demonstrate that the artifact generated from muscle stimulation can be suppressed at the input of the recording channel by using RTPPS.

The experiment setup for muscle stimulation is shown in Figure 9(a) [48]. The same chip configuration and ground/ power arrangement are used as in Figure 8(c). The recording needle electrode is inserted into the sciatic nerve on the left side of an anesthetized rat, and two concentric stimulation electrodes are inserted in the tibialis anterior (TIB) muscle of the right leg. Two concentric electrodes are put together and share the same reference voltage to form a tripolar stimulation electrode. The voltage waveforms on recording and stimulation electrodes are probed and observed using an oscilloscope. First of all, a biphasic stimulation pulse (amplitude $=90 \mu \mathrm{A}$, pulse width $=320 \mu \mathrm{s}$ ) is delivered to the muscle through WE1 and RE by activating SCG only. Figure 9(b) shows the output waveforms at RFE output. It is observed that the stimulation artifact is coupled to the input of RFE through the tissue and saturates its output. Figure 9(c) shows the RFE output when both SCG and CCG are enabled and tripolar stimulation is performed. The artifact is still observed. This is due to the asymmetry between the two electrode-tissue interfaces of WE1 and WE2. In Figure 9(d), the current amplitude generated from CCG is tuned (to a larger value in this case). To compensate the voltage on the recording site due to electrode asymmetry, the pulse width of CCG is divided into 16 steps and the current is different for each step. In the experiment, we tune the current amplitude of each step one by one by sending different commands to the DAC registers, until the artifact voltage cancellation is observed at the recording site. After the tuning, the stimulation artifact is significantly suppressed. Lastly, for comparison, in Figure 9(e), the reference voltage is disconnected from the tripolar electrode configuration to emulate the conventional push-pull bipolar stimulator [42]. In this case, we find that the artifact is a little smaller than the conventional bipolar stimulator, but still cannot be substantially suppressed due to the asymmetry of two stimulation interfaces. In conventional push-pull stimulators, the current tuning is inapplicable since there is no reference point for tuning. Therefore, the shared reference electrode must be present. In aforementioned experiments, successful muscle recruitment on the right foot of the rat is observed.

Another experiment setup for nerve stimulation and recording is shown Figure 9(f), when the system conducts concurrent neural stimulation and recording on the sciatic nerve of an anesthetized rat. The chips configuration and ground/power arrangement are the same as in Figure 8(c). Two concentric electrodes are tied together to form a tripolar (WE1, WE2, and RE) electrode and attached to the sciatic nerve. Note that these two concentric electrodes, however, may not be positioned well within the nerve cross section. This coarse arrangement leads to a possible asymmetric coupling between stimulation and recording sites, which results in asymmetric voltage waveforms at WE1 and WE2 for artifact suppression in this experiment. Two single-needle electrodes are used for recording. One recording electrode is inserted into the nerve which is about $5 \mathrm{~mm}$ away from the stimulation site, while the other one is placed in the animal body as a reference. Firstly, a stimulation pulse train is delivered to the nerve from the stimulator. For each pulse, the amplitude is set to $53 \mu \mathrm{A}$ and the pulse width is $320 \mu \mathrm{s}$ for both cathodic and anodic phases, with an interphasic delay of $25 \mu \mathrm{s}$. Foot dorsiflexion (FD) is observed, and evoked compound action potential (CAP) is recorded. In Figure $9(\mathrm{~g})$, the two test results with and without RTPPS are superimposed in the same graph. The top trace is the stimulation pulse waveform used in RTPPS mode. Middle trace is the recorded RFE response with RTPPS, and the bottom trace is the recorded REF response without RTPPS. Without RTPPS (CCG disabled), the large artifact is caused by saturation of the amplifier and DC voltage drift is observed at the RFE output. When RTPPS is enabled, the stimulation artifact is substantially suppressed. A series of evoked neural spikes can be clearly seen, and the amplitude of the suppressed artifact is 


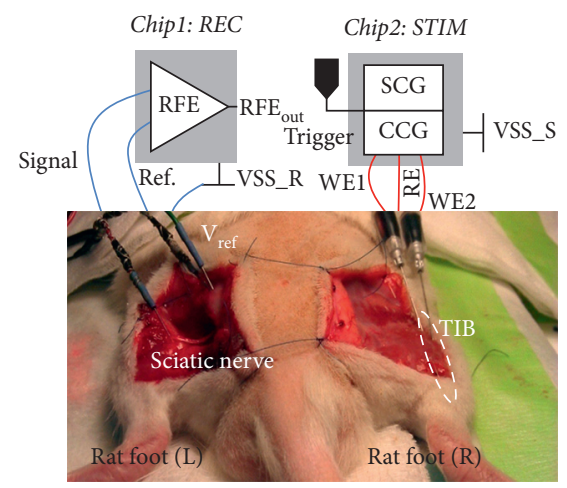

(a)

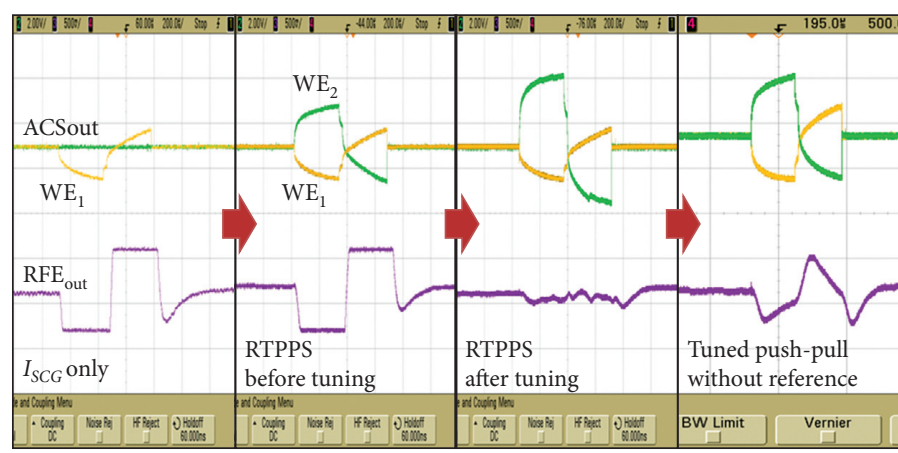

(b)

(d)

(e)

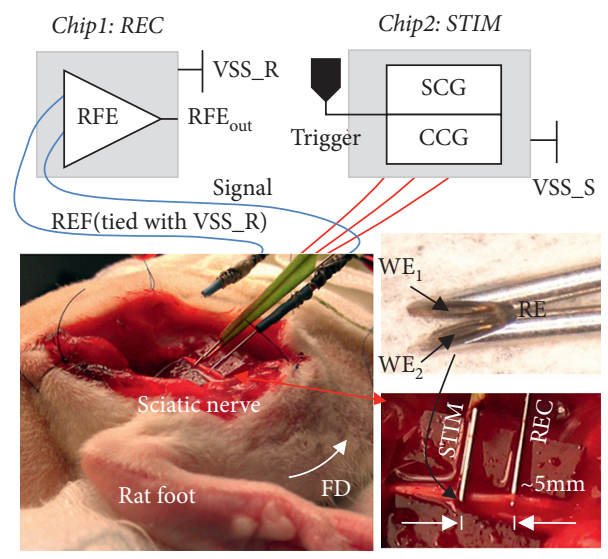

(f)

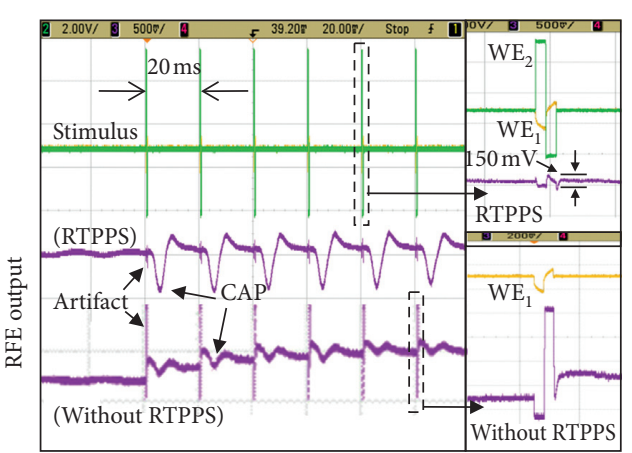

(g)

Figure 9: (a) In vivo test setup to observe muscle stimulation artifact suppression (recording in sciatic nerve). (b-e) Top traces are the stimulation pulse waveforms, and the bottom trace is the output from RFE. (f) In vivo test setup to observe neural stimulation artifact suppression and (g) test results including comparison to conventional bipolar stimulation.

TABLE 1: Comparison of stimulators for stimulation and recording.

\begin{tabular}{|c|c|c|c|c|c|c|c|}
\hline & & $\begin{array}{c}\text { Reference [49] } \\
\text { (REC and STIM) }\end{array}$ & $\begin{array}{c}\text { Reference [50] } \\
\text { (REC and STIM) }\end{array}$ & $\begin{array}{l}\text { Reference [37] } \\
\text { (REC and } \\
\text { STIM) }\end{array}$ & $\begin{array}{c}\text { Reference [40] } \\
\text { (REC and } \\
\text { STIM) }\end{array}$ & $\begin{array}{l}\text { Reference [24] } \\
\text { (REC and } \\
\text { STIM) }\end{array}$ & $\begin{array}{l}\text { This work } \\
\text { (REC and } \\
\text { STIM) }\end{array}$ \\
\hline \multicolumn{2}{|c|}{ Process } & $0.13 \mu \mathrm{m}$ & $65 \mathrm{~nm}$ & $40 / 0.18 \mu \mathrm{m} \mathrm{HV}$ & $0.13 \mu \mathrm{m}$ & $0.18 \mu \mathrm{m} \mathrm{HV}$ & $0.18 \mu \mathrm{m} \mathrm{HV}$ \\
\hline \multicolumn{2}{|c|}{ Artifact suppression } & $\begin{array}{l}\text { Differential } \\
\text { acquisition }\end{array}$ & $\begin{array}{l}\text { Digital adaptive } \\
\text { filter }\end{array}$ & ASAR & $\begin{array}{l}\text { Track-and- } \\
\text { zoom }\end{array}$ & Resetting RFE & RTPPS \\
\hline \multirow{5}{*}{ REC } & Supply voltage (V) & 1.2 & $1.2 / 2.5$ & $0.6 / 1.8 / 1.2 / \pm 5$ & $0.6 / 1.2 / 3.3$ & 1 & 1 \\
\hline & Gain $(\mathrm{dB})$ & - & 20 & - & 92 & 90 & $54 / 60$ \\
\hline & Input noise $(\mu$ Vrms $)$ & 2.6 & 2.9 & 2.2 & 1.6 & $71 \mathrm{nV} / \mathrm{rtHz}$ & 4.9 \\
\hline & NEF & 3.56 & - & - & 2.86 & 7.8 & 2.2 \\
\hline & $\begin{array}{l}\text { Power per channel } \\
(\mu \mathrm{W})\end{array}$ & 0.73 & 3.21 & 8.2 & 1.7 & 8 & 4.54 \\
\hline \multirow{4}{*}{ STIM } & $\begin{array}{l}\text { Compliance voltage } \\
\text { (V) }\end{array}$ & 3.3 & \pm 11 & \pm 8.5 & 3.3 & $3 / 6 / 9 / 12$ & 24 \\
\hline & Pulse amplitude (A) & - & $2 \mathrm{~m}$ & $20 \mu-5.1 \mathrm{~m}$ & $3 \mathrm{~m}$ & $5.04 \mathrm{~m}$ & $1.2 \mu-1.4 \mathrm{~m}$ \\
\hline & Pulse duration (s) & - & $10 \mu-2 \mathrm{~m}$ & $10 \mu-1.28 \mathrm{~m}$ & - & $15-500 \mu$ & $16 \mu-4 \mathrm{~m}$ \\
\hline & Pulse waveform & 8 bits, arbitrary & 8 bits, arbitrary & 8 bits, arbitrary & 8 bits, arbitrary & 6 bits, arbitrary & 5 bits, arbitrary \\
\hline
\end{tabular}

reduced to $80-150 \mathrm{mV}$ peak-to-peak, which is only $10 \%-$ $20 \%$ of the CAP signal recorded. The comparison of the prototype neural recording and stimulation chip to prior works is shown in Table 1. It can be found that we have achieved artifact suppression even with a high-gain recorder and large voltage compliance. 


\section{Conclusions}

A neural prosthesis chip with stimulation artifact suppression is presented in this work. The implemented 4-to-4 channel neural recording and stimulation system can be configured in recording, stimulation, recording-to-stimulation, and stimulation-to-recording modes, respectively. The function and efficacy of the proposed system has been demonstrated in both bench-top and in vivo experiments. The results show the stimulation induced artifact can be greatly suppressed during closed-loop neural recording and stimulation.

\section{Data Availability}

The data used to support the findings of this study are available from the corresponding author upon request.

\section{Disclosure}

The presentation of this manuscript is partly in the thesis for the degree of Doctor of Philosophy in Department of Electrical and Computer Engineering of NUS.

\section{Conflicts of Interest}

The authors declare that there are no conflicts of interest regarding the publication of this paper.

\section{Acknowledgments}

Thanks are due to the graduate program in National University of Singapore (NUS). This work was supported by the National Natural Science Foundation of China (NSFC) (Grant no. 61806012) and the Key Project of Science and Technology of Beijing Municipal Education Commission (Grant no. KZ201910005009).

\section{References}

[1] J.-I. Lee and M. Im, "Optimal electric stimulus amplitude improves the Selectivity between responses of ON versus OFF Types of retinal Ganglion cells," IEEE Transactions on Neural Systems and Rehabilitation Engineering, vol. 27, no. 10, pp. 2015-2024, 2019.

[2] K. Sooksood, E. Noorsal, J. Becker, and M. Ortmanns, "A neural stimulator front-end with arbitrary pulse shape, HV compliance and adaptive supply requiring $0.05 \mathrm{~mm}^{2}$ in $0.35 \mu \mathrm{m}$ HVCMOS," in Proceedings of the 2011 IEEE International Solid-State Circuits Conference, pp. 306-308, 2011.

[3] K. Chen, Z. Yang, L. Hoang, J. Weiland, M. Humayun, and W. Liu, "An integrated 256-channel Epiretinal prosthesis," IEEE Journal of Solid-State Circuits, vol. 45, no. 9, pp. 1946-1956, 2010.

[4] E. Noorsal, K. Sooksood, H. Xu, R. Hornig, J. Becker, and M. Ortmanns, "A neural stimulator Frontend with high-voltage compliance and programmable pulse shape for Epiretinal Implants," IEEE Journal of Solid-State Circuits, vol. 47, no. 1, pp. 244-256, 2012.

[5] L. Wagner, N. Maurits, B. Maat, D. Baskent, and A. E. Wagner, "The cochlear implant EEG artifact recorded from an artificial brain for Complex Acoustic Stimuli," IEEE Transactions on Neural Systems and Rehabilitation Engineering, vol. 26, no. 2, pp. 392-399, 2018.
[6] L. Rosenthal, D. Sweeney, A. L. Cunnington, L. R. Quinlan, and G. Ólaighin, "Sensory electrical stimulation Cueing may reduce Freezing of Gait Episodes in Parkinson's Disease," J Healthc Eng, vol. 2018, Article ID 4684925, 2018.

[7] M. Gharaei Jomehei and S. Sheikhaei, "A low-power lownoise CMOS bio-potential amplifier for multi-channel neural recording with active DC-rejection and current sharing," Microelectronics Journal, vol. 83, pp. 197-211, 2019.

[8] X. Yue, J. V. Huang, H. G. Krapp, and E. M. Drakakis, “An implantable mixed-signal CMOS die for battery-powered in vivo blowfly neural recordings," Microelectronics Journal, vol. 74, pp. 34-42, 2018.

[9] H. Ando, K. Takizawa, T. Yoshida, K. Matsushita, M. Hirata, and T. Suzuki, "Wireless multichannel neural recording with a 128-Mbps UWB transmitter for an implantable brain-machine interfaces," IEEE Trans Biomed Circuits Syst, vol. 10, pp. 1068-1078, 2016.

[10] R. Ramezani, Y. Liu, F. Dehkhoda et al., "On-probe neural interface ASIC for Combined electrical recording and Optogenetic stimulation," IEEE Trans Biomed Circuits Syst, vol. 12, pp. 576-588, 2018.

[11] J. D. Rolston, R. E. Gross, and S. M. Potter, "NeuroRighter: closed-loop multielectrode stimulation and recording for freely moving animals and cell cultures," Annu Int Conf IEEE Eng Med Biol Soc, pp. 6489-6492, 2009.

[12] H. T. Lancashire, D. Jiang, A. Demosthenous, and N. Donaldson, "An ASIC for recording and stimulation in Stacked Microchannel neural interfaces," IEEE Trans Biomed Circuits Syst, vol. 13, pp. 259-270, 2019.

[13] L. Carmona, P. F. Diez, E. Laciar, and V. Mut, "Multisensory stimulation and EEG recording below the Hair-Line: a New Paradigm on brain computer interfaces," IEEE Transactions on Neural Systems and Rehabilitation Engineering, vol. 28, pp. 825-831, 2020.

[14] Y. P. Xu, S. C. Yen, K. A. Ng, X. Liu, and T. C. Tan, “A Bionic Neural Link for peripheral nerve repair," Annu Int Conf IEEE Eng Med Biol Soc, pp. 1335-1338, 2012.

[15] S. Venkatraman, K. Elkabany, J. D. Long, Y. Yao, and J. M. Carmena, "A system for neural recording and closed-loop intracortical microstimulation in awake rodents," IEEE Transactions on Biomedical Engineering, vol. 56, pp. 15-22, 2009.

[16] J. Sacristán-Riquelme and M. T. Osés, "Implantable stimulator and recording device for artificial prosthesis control," Microelectronics Journal, vol. 38, pp. 1135-1149, 2007.

[17] C. Wei-Ming, C. Herming, C. Tsan-Jieh et al., "A fully integrated 8-channel closed-loop neural-prosthetic SoC for realtime epileptic seizure control," in IEEE International SolidState Circuits Conference Digest of Technical Papers2013, pp. 286-287, 2013.

[18] B. Zhang, W. Wang, S. Wang et al., "Clinical study on electronic medical Neuroelectric stimulation based on the Internet of Things to Treat Epilepsy Patients with Anxiety and Depression," J Healthc Eng, vol. 2021, p. 6667309, 2021.

[19] K. A. Ng, X. Liu, J. Zhao et al., "An inductively powered CMOS multichannel bionic neural link for peripheral nerve function restoration," in IEEE Asian Solid State Circuits Conference (A-SSCC), pp. 181-184, 2012.

[20] A. Asfour, C. Fiche, and C. Deransart, "Dedicated electronics for electrical stimulation and EEG recording using the same electrodes: application to the automatic control of epileptic seizures by neurostimulation," in EEE Instrumentation \& $\mathrm{Mea}$ surement Technology Conference IMTC 20072007, pp. 1-4, I, 2007.

[21] C. Y. Lin, W. L. Chen, and M. D. Ker, "Implantable stimulator for epileptic seizure suppression with loading impedance 
adaptability," IEEE Trans Biomed Circuits Syst, vol. 7, pp. 196-203, 2013.

[22] T.-J. Chen, C. Jeng, S.-T. Chang et al., "A hardware implementation of real-time epileptic seizure detector on FPGA," in 2011 IEEE Biomedical Circuits and Systems Conference (BioCAS), pp. 25-28, 2011.

[23] H. Jeon, J.-S. Bang, Y. Jung, I. Choi, and M. Je, "A high DR, DC-coupled, time-based neural-recording IC with Degeneration R-DAC for bidirectional neural interface," IEEE Journal of Solid-State Circuits, vol. 54, pp. 2658-2670, 2019.

[24] B. C. Johnson, S. Gambini, I. Izyumin et al., "An implantable $700 \mu \mathrm{W}$ 64-channel neuromodulation IC for simultaneous recording and stimulation with rapid artifact recovery," in 2017 Symposium on VLSI Circuits, pp. C48-C49, 2017.

[25] M. R. Pazhouhandeh, H. Kassiri, A. Shoukry, I. Wesspapir, P. Carlen, and R. Genov, "Artifact-tolerant Opamp-Less Delta-Modulated bidirectional neuro-interface," in IEEE Symposium on VLSI Circuits2018, pp. 127-128, 2018.

[26] J. P. Uehlin, W. A. Smith, V. R. Pamula et al., "A single-chip bidirectional neural interface with high-voltage stimulation and adaptive artifact cancellation in Standard CMOS," IEEE Journal of Solid-State Circuits, vol. 55, pp. 1749-1761, 2020.

[27] K. C. McGill, K. L. Cummins, L. J. Dorfman et al., "On the nature and elimination of stimulus artifact in nerve signals evoked and recorded using surface electrodes," IEEE Transactions on Biomedical Engineering, vol. 29, pp. 129-137, 1982.

[28] K. Yoshida and K. Horch, "Closed-loop control of ankle position using muscle afferent feedback with functional neuromuscular stimulation," IEEE Transactions on Biomedical Engineering, vol. 43, pp. 167-176, 1996.

[29] R. A. Blum, J. D. Ross, E. A. Brown, and S. P. DeWeerth, “An integrated system for simultaneous, multichannel neuronal stimulation and recording," IEEE Transactions on Circuits and Systems I: Regular Papers, vol. 54, pp. 2608-2618, 2007.

[30] M. Mc Laughlin, T. Lu, A. Dimitrijevic, and F. G. Zeng, "Towards a closed-loop cochlear implant system: application of embedded monitoring of peripheral and central neural activity," IEEE Transactions on Neural Systems and Rehabilitation Engineering, vol. 20, pp. 443-454, 2012.

[31] A. R. Kent and W. M. Grill, "Instrumentation to record evoked potentials for closed-loop control of deep brain stimulation," Annu Int Conf IEEE Eng Med Biol Soc, pp. 6777-6780, 2011.

[32] F. B. Myers, O. J. Abilez, C. K. Zarins, and L. P. Lee, "Stimulation and artifact-free extracellular electrophysiological recording of cells in suspension," Annu Int Conf IEEE Eng Med Biol Soc, pp. 4030-4033, 2011.

[33] X. Yi, J. Jia, S. Deng, S. G. Shen, Q. Xie, and G. Wang, "A blink restoration system with contralateral EMG triggered stimulation and real-time artifact blanking," IEEE Trans Biomed Circuits Syst, vol. 7, pp. 140-148, 2013.

[34] B. Bozorgzadeh, D. P. Covey, C. D. Howard, P. A. Garris, and P. Mohseni, "A Neurochemical pattern generator SoC with switched-electrode Management for single-chip electrical stimulation and $9.3 \mu \mathrm{W}, 78 \mathrm{pA} \mathrm{rms}, 400 \mathrm{~V} / \mathrm{s}$ FSCV sensing," IEEE Journal of Solid-State Circuits, vol. 49, pp. 881-895, 2014.

[35] A. E. Mendrela, J. Cho, J. A. Fredenburg, C. A. Chestek, M. P. Flynn, and E. Yoon, "Enabling closed-loop neural interface: a bi-directional interface circuit with stimulation artifact cancellation and cross-channel CM noise suppression," in 2015 Symposium on VLSI Circuits (VLSI Circuits), pp. C108-C109, 2015.

[36] A. E. Mendrela, J. Cho, and J. A. Fredenburg, "A bidirectional neural interface circuit with active stimulation artifact cancellation and cross-channel Common-mode noise suppression," IEEE Journal of Solid-State Circuits, vol. 51, pp. 955-965, 2016.

[37] D. Rozgic, V. Hokhikyan, W. Jiang et al., “A $0.338 \mathrm{~cm}(3)$, artifact-free, 64-Contact neuromodulation Platform for simultaneous stimulation and sensing," IEEE Trans Biomed Circuits Syst, vol. 13, pp. 38-55, 2019.

[38] H. Deprez, R. Gransier, M. Hofmann, A. van Wieringen, J. Wouters, and M. Moonen, "Template subtraction to remove CI stimulation artifacts in Auditory Steady-state responses in CI Subjects," IEEE Transactions on Neural Systems and Rehabilitation Engineering, vol. 25, pp. 1322-1331, 2017.

[39] M. Yochum and S. Binczak, "A wavelet based method for electrical stimulation artifacts removal in electromyogram," Biomed Signal Proces, vol. 22, pp. 1-10, 2015.

[40] M. Reza Pazhouhandeh, M. Chang, T. A. Valiante, and R. Genov, "Track-and-Zoom neural analog-to-digital converter with Blind stimulation artifact rejection," IEEE Journal of Solid-State Circuits, vol. 55, pp. 1984-1997, 2020.

[41] W. Jiang, V. Hokhikyan, H. Chandrakumar, V. Karkare, and D. Markovic, "A \pm 50 - $\mathrm{mV}$ Linear-input-range VCO-based neural-recording front-end with digital Nonlinearity correction," IEEE Journal of Solid-State Circuits, vol. 52, pp. 173-184, 2017.

[42] Y. T. Wong, N. Dommel, P. Preston et al., "Retinal neurostimulator for a multifocal vision prosthesis," IEEE Transactions on Neural Systems and Rehabilitation Engineering, vol. 15, pp. 425-434, 2007.

[43] C. Yung-Chan, L. Yu-Tao, S.-R. Yeh, and C. Hsin, "A bidirectional, flexible neuro-electronic interface employing localised stimulation to reduce artifacts," in International IEEE/EMBS Conference on Neural Engineering2009, pp. 4650, 2009 4th.

[44] S. Nag, S. K. Sikdar, N. V. Thakor, V. R. Rao, and D. Sharma, "Sensing of stimulus artifact suppressed signals from electrode interfaces," IEEE Sensors Journal, vol. 15, pp. 3734-3742, 2015.

[45] L. Xu, Y. Lei, L. Peng et al., "An artifact-suppressed stimulator for simultaneous neural recording and stimulation systems," Annu Int Conf IEEE Eng Med Biol Soc, pp. 2118-2121, 2017.

[46] L. Liu, X. Zou, W. L. Goh, R. Ramamoorthy, G. Dawe, and M. Je, " $\mathrm{nW} 43 \mathrm{nV} /$ root $\mathrm{Hz}$ neural recording amplifier with enhanced noise efficiency factor," Electronics Letters, vol. 48, pp. 479-U426, 800.

[47] X. Zou, L. Liu, J. H. Cheong et al., "A 100-channel 1-mW implantable neural recording IC," IEEE Transactions on Circuits and Systems I: Regular Papers, vol. 60, pp. 2584-2596, 2013.

[48] X. Liu, Design of Integrated Neural/Modular Stimulators, [Doctoral thesis, National University of Singapore], ScholarBank@NUS Repository, 2014.

[49] M. R. Pazhouhandeh, H. Kassiri, A. Shoukry, I. Wesspapir, P. Carlen, and R. Genov, "Artifact-tolerant Opamp-Less Delta-Modulated bidirectional neuro-interface," in IEEE Symposium on VLSI Circuits2018, pp. 127-128, 2018.

[50] J. P. Uehlin, W. A. Smith, V. R. Pamula et al., "A single-chip bidirectional neural interface with high-voltage stimulation and adaptive artifact cancellation in Standard CMOS," IEEE Journal of Solid-State Circuits, vol. 55, pp. 1749-1761, 2020. 